\title{
Percepção de universitários de uma instituição de ensino superior do Alto Sertão da Paraíba - Brasil a respeito de agravos sistêmicos frente a infecções odontogênicas
} Perception of undergraduate at university of Sertão of Paraíba - Brazil regarding systemic impairment of odontogenic infections

Percepción de los estudiantes universitarios do Sertão da Paraíba - Brasil con respecto a los comprometimientos sistémicos de las infecciones odontogénicas

Tharley GOMES

DDS, Graduando, Departamento de Odontologia da Faculdade Santa Maria, FSM, 58900-000 Cajazeiras - PB, Brasil Marcos Alexandre Casimiro OLIVEIRA MS, Professor, Departamento de Odontologia da Faculdade Santa Maria (FSM) 58900-000 Cajazeiras - PB, Brasil https://orcid.org/0000-0002-7308-6779 Raulison Vieira SOUSA PhD, Professor, Departamento de Odontologia da Faculdade Santa Maria, FSM, 58900-000 Cajazeiras - PB, Brasil https://orcid.org/0000-0003-1106-5259 Luan Éverton Galdino BARNABÉ Doutorando, Universidade Estadual da Paraíba, UEPB, 58429-500, Campina-Grande - PB, Brasil https://orcid.org/0000-0001-9657-5180 José Klidenberg de OLIVEIRA JúNIOR MS, Professor, Departamento de Odontologia da Faculdade Santa Maria (FSM) 58900-000 Cajazeiras - PB, Brasil https://orcid.org/0000-0002-4539-2007

\section{Resumo}

As infecções odontogênicas são processos causados por cárie dentária, infecções endodônticas, pericoronarite e doenças periodontais. Em casos evoluídos, pode ocorrer a disseminação para regiões importantes do corpo. O objetivo do presente estudo foi avaliar a percepção de universitários de uma Instituição de Ensino Superior (IES) do alto sertão paraibano sobre a evolução das infecções odontogênicas. O trabalho em questão refere-se a um estudo transversal, de natureza descritiva e quantitativa, o qual foi realizado com os universitários matriculados na Faculdade Santa Maria, de todos os cursos de graduação (exceto Odontologia) e de todos os períodos. Foi realizado por meio de um questionário on-line estruturado, aplicado através da plataforma Google Forms e composto de três etapas. Foram entrevistados 143 universitários de ambos os sexos, entre as idades de 18 a 54 anos, sendo a maior parte mulheres $(83,9 \%)$ e a idade mais prevalente compreende os indivíduos entre os 18 a 30 anos (85\%). Em relação ao conhecimento sobre cárie dentária, 97,2\% responderam que conheciam a doença. Quando indagados sobre ao entendimento de como evitar a cárie e a gengivite, $77,6 \%$ dos alunos responderam que sabiam métodos para evitar o surgimento das doenças. Mais da metade dos alunos $(55,9 \%)$ responderam que concordavam totalmente que a cárie dentária e um canal não tratado poderiam ocasionar a formação de conteúdo purulento. Os resultados sugerem que os alunos da Faculdade Santa Maria possuem um bom conhecimento relacionado a infecções odontogênicas e seus agravos. Porém, esse conhecimento é limitado, mostrando assim que o mesmo deve ser aprofundado.

Descritores: Educação em Saúde; Infecções; Odontologia.

\section{Abstract}

Odontogenic infections are processes caused by tooth decay, endodontic infections, pericoronitis and periodontal diseases. In advanced cases, it can spread to important regions of the body. The aim of the present study was to evaluate the perception of university students from a Higher Education Institution (HEI) in the highlands of Paraíba about the evolution of odontogency infections. The work in question refers to a cross-sectional study, of a descriptive and quantitative nature, which was carried out with university students enrolled at Faculdade Santa Maria, from all undergraduate courses (except Dentistry) and from all periods. It was carried out through a structured online questionnaire, applied through the Google Forms platform and composed of three stages. 143 university students of both sexes were interviewed, between the ages of 18 to 54 years old, most of them women (83.9\%) and the most prevalent age comprises individuals between 18 to 30 years old (85\%). Regarding knowledge about dental caries, $97.2 \%$ answered that they knew about the disease. When asked about understanding how to prevent cavities and gingivitis, $77.6 \%$ of students answered that they knew methods to prevent the onset of diseases. More than half of the students (55.9\%) replied that they totally agreed that dental caries and an untreated canal could cause the formation of purulent content. The results suggest that students at Faculdade Santa Maria have good knowledge related to odontogenic infections and their problems. However, this knowledge is limited, thus showing that it must be deepened.

Descriptors: Health Education; Infections; Dentistry.

\section{Resumen}

Las infecciones odontogénicas son procesos provocados por caries, infecciones endodónticas, pericoronitis y enfermedades periodontales. En casos avanzados, puede extenderse a regiones importantes del cuerpo. El objetivo del presente estudio fue evaluar la percepción de estudiantes universitarios de una Institución de Educación Superior (IES) en el altiplano de Paraíba sobre la evolución de las infecciones odontogénicas. El trabajo en cuestión se refiere a un estudio transversal, de carácter descriptivo y cuantitativo, que se llevó a cabo con estudiantes universitarios matriculados en la Faculdade Santa María, de todas las carreras (excepto Odontología) y de todos los períodos. Se llevó a cabo mediante un cuestionario online estructurado, aplicado a través de la plataforma Google Forms y compuesto por tres etapas. Se entrevistaron 143 estudiantes universitarios de ambos sexos, con edades comprendidas entre los 18 y los 54 años, la mayoría mujeres (83,9\%) y la edad más prevalente corresponde a los individuos entre 18 y 30 años (85\%). En cuanto al conocimiento sobre la caries dental, el $97,2 \%$ respondió que conocía la enfermedad. Cuando se les preguntó sobre cómo prevenir la caries y la gingivitis, el $77,6 \%$ de los estudiantes respondió que conocía métodos para prevenir la aparición de enfermedades. Más de la mitad de los estudiantes (55,9\%) respondieron que estaban totalmente de acuerdo en que la caries dental y un canal no tratado podrían provocar la formación de contenido purulento. Los resultados sugieren que los estudiantes de la Faculdade Santa Maria tienen buenos conocimientos relacionados con las infecciones odontogénicas y sus problemas. Sin embargo, este conocimiento es limitado, lo que demuestra que debe profundizarse.

Descriptores: Educación para la Salud; Infecciones; Odontología.

INTRODUÇÃO

As infecções odontogênicas são processos causados por cárie dentária, infecção endodôntica, pericoronarite e doenças periodontais. Essas infecções iniciam-se em um dente ou tecido isoladamente, porém, se o paciente negligenciar e não procurar tratamento odontológico, a mesma pode se disseminar por tecidos vizinhos ou causar complicações sistêmicas ${ }^{1}$. 
Entre as formas etiológicas citadas, a infecção endodôntica é a mais comum dentre as outras e pode ser diferenciada em três tipos: a infecção endodôntica primária, causada pela necrose pulpar, a infecção endodôntica secundária que surge durante/após 0 tratamento endodôntico, e a infecção endodôntica persistente, onde os microorganismos da infecção primária e secundária irão persistir ao preparo químico-mecânico e a obturação. A evolução dessas condições consiste na infecção endodôntica extraradicular, sendo caracterizada pela disseminação da infecção para os tecidos da região perriradicular².

Embora essas infecções odontogênicas sejam de fácil resolução quando diagnosticadas no início, elas podem evoluir para quadros clínicos graves e se disseminar para outros tecidos do corpo podendo envolver espaços faciais da cabeça e pescoço, músculos mastigatórios, ossos, veias, artérias, orbita, seio cavernoso e mediastino ${ }^{3}$.Quando essa disseminação ocorre, 0 paciente pode apresentar muitas complicações graves, como obstrução das vias aéreas, trombose do seio cavernoso, mediastinite, endocardite e abscesso cerebral ${ }^{4}$.

Umas das principais e mais graves infecções odontogênicas complexas é a Angina de Ludwig. Essa condição é caracterizada pela disseminação da infecção para o espaço submandibular, sublingual e submentual, causando várias complicações que podem comprometer a vida do paciente, como por exemplo: obstrução das vias aéreas, ruptura da artéria carótida, mediastinite, derrame pericárdio e pleural, osteomielite e pneumonia por aspiração ${ }^{5}$.

As infecções odontogênicas complexas atingem pessoas de diferentes idades e não há predileção pelo gênero dos pacientes. Entre os fatores que causam a evolução da doença, os socioeconômicos têm sua ligação, pois nas pesquisas analisadas, os pacientes participantes tinham renda e nível de escolaridade baixos. A negligência também é um fator, visto que a maioria dos pacientes que desenvolveram essas infecções complexas, não relaram idas ao dentista para exames de rotina ${ }^{5}$. A educação em saúde também se mostra como um processo relevante para a evolução ou não de uma doença, pois integra a população na busca da saúde, como também garante certa autonomia para cuidados com a própria higiene bucal $^{6 .}$

$\mathrm{Na}$ Odontologia, o conhecimento sobre educação em saúde é algo de fundamental importância na vida do paciente, visto que pode proporcionar benefícios a saúde bucal e sistêmica. Isso ocorre, porque o conhecimento acerca de práticas preventivas, que muitas vezes são desconhecidas pelo paciente, pode se tornar decisivas para a qualidade de vida desse indivíduo ${ }^{7}$

Levando em consideração a importância e os riscos das infecções odontogênicas e seus agravos, torna-se muito relevante realizar uma pesquisa para analisar o nível de conhecimento dos universitários matriculados na Faculdade Santa Maria, de todos os cursos de graduação, exceto Odontologia, e também de todos os períodos, em relação a essas doenças, buscando entender se o processo evolutivo das mesmas está atrelado a falhas no processo de educação em saúde e falta de informação. Portanto, o objetivo desse presente trabalho foi avaliar a percepção de universitários de uma Instituição de Ensino Superior (IES) localizada em um município de médio porte sobre a evolução das infecções odontogênicas.

MATERIAL E MÉTODO

- Tipo de pesquisa

O trabalho em questão refere-se a um estudo transversal, de natureza descritiva e quantitativa, através da técnica de documentação direta extensiva, utilizando um questionário on-line ${ }^{8}$.O principal foco foi identificar através de perguntas estruturadas qual o conhecimento dos universitários matriculados na Faculdade Santa Maria, de todos os cursos de graduação (exceto Odontologia) e de todos os períodos, a respeito de agravos de infecções odontogênicas.

- Local e período da pesquisa

Com uma população estimada em 61.993 habitantes, o município de Cajazeiras, na Paraíba, tem uma área total de 565,899 km2 e é um dos principais polos de educação de ensino superior do interior da Paraíba. O município sedia campus da Universidade Federal de Campina Grande -UFCG, do Instituto Federal da Paraíba - IFPB, bem como Instituições de Ensino Superior Privado, a exemplo da Faculdade Santa Maria - FSM. Do ponto de vista geográfico, a cidade de Cajazeiras localiza-se em uma região estratégica na Paraíba por sua proximidade com estados vizinhos, como Ceará e Rio Grande do Norte. Em consequência, é destino de escolha para a graduação do ensino superior por muitos discentes. A pesquisa foi realizada na Faculdade Santa Maria (FSM), em Cajazeiras, Paraíba, Brasil, após a anuência da instituição. A pesquisa iniciou-se em outubro de 2020 e se encerrou em novembro do mesmo ano, tendo assim uma duração de 2 meses de pesquisa. 


\section{- População e amostra}

A pesquisa selecionou uma população composta por universitários regularmente matriculados na Faculdade Santa Maria e essa informação foi concedida através da solicitação da listagem de alunos na secretaria acadêmica. A amostra inclui todos os universitários matriculados nos cursos de graduação da Faculdade Santa Maria, exceto os acadêmicos de odontologia para evitar possíveis víeis no estudo.

\section{- Tamanho da amostra}

O tamanho da amostra foi por conveniência de forma não probabilística e não aleatória. Foram selecionados 143 universitários que consentiram participar da pesquisa e assinaram o TCLE e que estavam dentro dos critérios de inclusão.

\section{- Aspectos éticos}

Essa pesquisa segue as normas explanadas na Resolução 510/16 do Conselho Nacional de Saúde e foi submetida à análise do comitê de Ética em pesquisa com seres humanos da Faculdade Santa Maria (FSM), onde foi aprovado e consta com o número de parecer 4.365.747.

Os sujeitos da pesquisa foram informados com antecedência a respeito dos objetivos e procedimentos do estudo, como também da confiabilidade dos dados registrados e do anonimato da colaboração dos mesmos. Posteriormente, foi solicitado ao participante que assinasse ou registrasse a impressão digital no documento termo de Consentimento Livre e Esclarecido (de acordo com a Resolução $\mathrm{n}^{\circ}$ 510/16 do Conselho Nacional de Saúde/MS, sobre Pesquisa envolvendo seres humanos.

\section{- Critérios de inclusão e exclusão}

Os critérios de inclusão para a pesquisa são homens e mulheres, adultos e idosos, entre as idades de 18 a 70 anos, que são matriculados na Faculdade Santa Maria durante o período da coleta de dados, que não cursam Odontologia e que assinarem o TCLE. Os critérios de exclusão são: universitários que não souberem e/ou tiverem dificuldade de utilizar a plataforma on-line para responder 0 questionário, universitários que responderem de forma errada os questionários e os universitários que não colaborarem com os prazos para responder a pesquisa.

- Instrumentos de coletas de dados

A coleta de dados foi feita através de um questionário on-line elaborado através da plataforma Google Forms. O questionário em questão foi retirado da pesquisa de Pinheiro et al 2005, composta de três partes, contendo perguntas claras e objetivas com respostas de múltiplas escolhas, as quais tiveram o intuito de avaliar o perfil socioeconômico dos entrevistados, avaliar o conhecimento desses universitários matriculados na Faculdade Santa Maria e também avaliar o nível de acesso a informação referente a saúde bucal. $O$ questionário foi enviado para o e-mail e WhatsApp dos universitários que aceitaram participar da pesquisa.

Previamente à aplicação do questionário foi realizado um estudo piloto com 10 universitários $(n=10)$ onde verificou-se que o questionário apresenta fidedignidade (qualquer pessoa que 0 aplique obterá sempre os mesmos resultados), validade (os dados recolhidos são necessários à pesquisa) e operatividade (vocabulário acessível e significado claro).

\section{- Procedimento de coleta de dados}

Os dados foram coletados através da plataforma on-line Google Forms. Participaram os universitários que aceitaram participar da pesquisa e assinaram digitalmente o Termo de Consentimento Livre e Esclarecido, que fizeram parte dos critérios de inclusão e que tiveram capacidade de utilizar a plataforma on-line para responder o questionário.

O questionário foi encaminhado para o e-mail e WhatsApp dos universitários que aceitaram participar da pesquisa. $\mathrm{O}$ arquivo foi enviado três vezes pela mesma via: primeiro no momento em que a pesquisa se iniciou, o segundo no período de duas semanas após a data do primeiro envio e o terceiro no período de três semanas após a data do primeiro envio. A ausência de respostas do universitário foi lida como não adesão ao presente estudo, sendo automaticamente substituído com o intuito de não causar problemas na amostra.

\section{- Processamento e análise de dados}

Os dados foram apresentados através de tabelas e um gráfico, onde foi possível ver porcentagens referentes a cada uma das perguntas do questionário. Também foi aplicado o teste-t independente no programa SPSS 22.0 (SPSS Inc., EUA), onde foi avaliado e comparado a média de acertos entre cada grupo, como por exemplo mulheres e homens. Primeiramente, definiu-se a pontuação da segunda parte do questionário, o qual é de 13 pontos, visto que são 13 questões focadas na avaliação do conhecimento dos universitários (segunda parte do questionário). Os alunos conseguiam o ponto quando respondiam "sim" ou "concordo totalmente" nas perguntas.

RESULTADOS

Foram entrevistados 143 universitários de ambos os sexos, entre as idades de 18 a 54 
anos. A maior parte da amostra é composta por mulheres $(83,3 \%)$ e a idade mais prevalente compreende os indivíduos entre os 18 a 30 anos de idade (88,5\%). Em relação a etnia, notou-se que a maior parte dos entrevistados se categorizam como pardos $(47,6 \%)$, a renda mensal predominante foi de um salário mínimo $(35,7 \%)$ e a quantidade de pessoas que vivem na casa da maioria dos entrevistados foi de 3 a 4 pessoas $(70,4 \%)$. No que concerne ao curso de graduação, universitários do curso de enfermagem foram os que mais participaram da pesquisa, totalizado $25,2 \%$ dos entrevistados. $O$ estado civil mais prevalente foi o de solteiro, correspondendo a $81,8 \%$ (Tabela 1).

Tabela 1. Descrição do perfil socioeconômico dos universitários entrevistados $(n=143)$, Cajazeiras - PB, Brasil

\begin{tabular}{|c|c|c|c|}
\hline Variáveis & Alternativas & Quantidade (n) & Porcentagem \\
\hline \multirow{2}{*}{ Gênero } & Feminino & 120 & $83,3 \%$ \\
\hline & Masculino & 23 & $16,7 \%$ \\
\hline \multirow{4}{*}{ Idade } & $18-30$ & 129 & $88,5 \%$ \\
\hline & $31-40$ & 10 & $8,45 \%$ \\
\hline & $41-50$ & 03 & $2,8 \%$ \\
\hline & Acima de 50 & 01 & $0,7 \%$ \\
\hline \multirow{4}{*}{ Etnia } & Branco & 67 & $46,9 \%$ \\
\hline & Negro & 08 & $5,6 \%$ \\
\hline & Pardo & 68 & $47,6 \%$ \\
\hline & Indígena & 00 & - \\
\hline \multirow{5}{*}{ Média de renda } & $\begin{array}{l}1 \\
\text { mínimo }\end{array}$ & 51 & $35,7 \%$ \\
\hline & $\begin{array}{l}2 \text { salários } \\
\text { mínimos }\end{array}$ & 43 & $30,1 \%$ \\
\hline & $\begin{array}{l}3 \text { a } 5 \text { salários } \\
\text { mínimos }\end{array}$ & 35 & $24,5 \%$ \\
\hline & $\begin{array}{l}6 \text { a } 10 \text { salários } \\
\text { mínimos }\end{array}$ & 12 & $8,4 \%$ \\
\hline & $\begin{array}{ll}\text { Acima de } & 10 \\
\text { salários } & \\
\text { mínimos } & \end{array}$ & 2 & $1,4 \%$ \\
\hline \multirow{4}{*}{$\begin{array}{l}\text { Quantas pessoas } \\
\text { vivem na casa }\end{array}$} & $1-2$ & 20 & $14 \%$ \\
\hline & $3-4$ & 101 & $70,5 \%$ \\
\hline & 5 & 13 & $9,15 \%$ \\
\hline & Mais de 5 & 9 & $6,3 \%$ \\
\hline \multirow{10}{*}{$\begin{array}{l}\text { Curso } \\
\text { graduaçãa }\end{array}$} & Farmácia & 16 & $11,2 \%$ \\
\hline & $\begin{array}{ll}\begin{array}{l}\text { Arquitetura } \\
\text { urbanismo }\end{array} & \mathrm{e} \\
\end{array}$ & 12 & $8,4 \%$ \\
\hline & Medicina & 7 & $4,9 \%$ \\
\hline & $\begin{array}{l}\text { Engenharia } \\
\text { civil }\end{array}$ & 6 & $4,2 \%$ \\
\hline & Enfermagem & 36 & $25,2 \%$ \\
\hline & Fisioterapia & 11 & $7,7 \%$ \\
\hline & Biomedicina & 11 & $7,7 \%$ \\
\hline & Nutrição & 10 & $7,0 \%$ \\
\hline & Psicologia & 31 & $21,8 \%$ \\
\hline & Administração & 4 & $2,8 \%$ \\
\hline \multirow{5}{*}{ Estado civil } & Solteiro & 117 & $81,8 \%$ \\
\hline & Casado & 20 & $14 \%$ \\
\hline & Viúvo & 0 & $0.0 \%$ \\
\hline & Divorciado & 3 & $2,1 \%$ \\
\hline & Outro & 3 & $2,1 \%$ \\
\hline
\end{tabular}

A segunda parte do questionário foi responsável por analisar o nível de conhecimento dos universitários a respeito das infecções odontogênicas, contendo um total de 13 perguntas e respectivamente, 13 acertos. A primeira pergunta indagava inicialmente se os universitários tinham conhecimento sobre o que era uma cárie dentária, onde a maior parte deles respondeu que conhecia a doença $(97,2 \%)$. Em contrapartida, quando foi perguntado em relação ao conhecimento sobre o que é uma gengivite e um tratamento de canal, essa porcentagem caiu, mostrando que $88,1 \%$ dos entrevistados sabiam o que é uma gengivite e apenas $65 \%$ sabiam o que é um tratamento de canal (Tabela 2).
Tabela 2. Descrição das respostas do segundo questionário $(n=143)$, Cajazeiras - PB, Brasil

\begin{tabular}{|c|c|c|c|}
\hline Variáveis & Alternativas & Quantidade (n) & Porcentagem \\
\hline \multirow{2}{*}{$\begin{array}{l}\text { Você sabe o que é uma cárie } \\
\text { dentária? }\end{array}$} & Sim & 139 & $97,2 \%$ \\
\hline & Não & 4 & $2,8 \%$ \\
\hline \multirow[b]{2}{*}{$\begin{array}{l}\text { Você sabe o que é uma } \\
\text { gengivite? }\end{array}$} & Sim & 126 & $88,1 \%$ \\
\hline & Não & 17 & $11,9 \%$ \\
\hline \multirow[b]{2}{*}{$\begin{array}{l}\text { Você sabe o que é um } \\
\text { tratamento de canal? }\end{array}$} & Sim & 93 & $65 \%$ \\
\hline & Não & 50 & $35 \%$ \\
\hline \multirow[b]{2}{*}{$\begin{array}{l}\text { Você possui conhecimento } \\
\text { sobre como evitar a cárie } \\
\text { dentária ou gengivite? }\end{array}$} & Sim & 111 & $77,6 \%$ \\
\hline & Não & 32 & $22,4 \%$ \\
\hline \multirow{2}{*}{$\begin{array}{l}\text { Você já recebeu alguma } \\
\text { informação sobre a cárie } \\
\text { dentáriar gengivite, } \\
\text { tratamento de canal ou } \\
\text { outras doenças/condições } \\
\text { bucais? }\end{array}$} & Sim & 95 & $66,4 \%$ \\
\hline & Não & 48 & $33,6 \%$ \\
\hline \multirow{5}{*}{$\begin{array}{l}\text { Você concorda que uma } \\
\text { cárie dentária e um canal } \\
\text { não tratado podem causar } \\
\text { formação de pus? }\end{array}$} & $\begin{array}{l}\text { Concordo } \\
\text { totalmente }\end{array}$ & 80 & $55,9 \%$ \\
\hline & \begin{tabular}{|l|l}
$\begin{array}{l}\text { Concordo } \\
\text { parcialmente }\end{array}$ \\
\end{tabular} & 21 & $14,7 \%$ \\
\hline & \begin{tabular}{|l} 
Discordo \\
totalmente
\end{tabular} & 3 & $2,1 \%$ \\
\hline & \begin{tabular}{|l|l} 
Discordo \\
parcialmente
\end{tabular} & 3 & $2,1 \%$ \\
\hline & Não sei responder & 36 & $25,2 \%$ \\
\hline \multirow{5}{*}{$\begin{array}{l}\text { Você concorda que uma } \\
\text { cárie dentária, gengivite e } \\
\text { um canal não tratado } \\
\text { podem evoluir para } \\
\text { grandes infecções e causar } \\
\text { problemas graves no nosso } \\
\text { corpo? }\end{array}$} & $\begin{array}{l}\text { Concordo } \\
\text { totalmente }\end{array}$ & 105 & $73,4 \%$ \\
\hline & $\begin{array}{l}\begin{array}{l}\text { Concordo } \\
\text { parcialmente }\end{array} \\
\end{array}$ & 10 & $7 \%$ \\
\hline & \begin{tabular}{|l} 
Discordo \\
totalmente
\end{tabular} & 3 & $2,1 \%$ \\
\hline & \begin{tabular}{|l|} 
Discordo \\
parcialmente
\end{tabular} & 4 & $2,8 \%$ \\
\hline & Não sei responder & 21 & $14,7 \%$ \\
\hline \multirow{5}{*}{$\begin{array}{l}\text { Você concorda que uma } \\
\text { cárie dentária, gengivite } \\
\text { um canal não tratado } \\
\text { podem evoluir para um } \\
\text { quadro de internação } \\
\text { hospitalar? }\end{array}$} & $\begin{array}{l}\text { Concordo } \\
\text { totalmente }\end{array}$ & 87 & $60,8 \%$ \\
\hline & \begin{tabular}{|l}
$\begin{array}{l}\text { Concordo } \\
\text { parcialmente }\end{array}$ \\
\end{tabular} & 18 & $12,6 \%$ \\
\hline & $\begin{array}{l}\text { Discordo } \\
\text { totalmente }\end{array}$ & 4 & $2,8 \%$ \\
\hline & \begin{tabular}{|l|} 
Discordo \\
parcialmente
\end{tabular} & 2 & $1,4 \%$ \\
\hline & Não sei responder & 32 & $22,4 \%$ \\
\hline \multirow{5}{*}{$\begin{array}{l}\text { Você concorda que uma } \\
\text { cárie dentária, gengivite e } \\
\text { um canal não tratado } \\
\text { podem evoluir e causar a } \\
\text { falta/dificuldade } \\
\text { respiração? }\end{array}$} & $\begin{array}{l}\text { Concordo } \\
\text { totalmente }\end{array}$ & 44 & $30,8 \%$ \\
\hline & $\begin{array}{l}\begin{array}{l}\text { Concordo } \\
\text { parcialmente }\end{array} \\
\end{array}$ & 18 & $12,6 \%$ \\
\hline & \begin{tabular}{|l|l}
$\begin{array}{l}\text { Discordo } \\
\text { totalmente }\end{array}$ \\
\end{tabular} & 4 & $2,8 \%$ \\
\hline & \begin{tabular}{|l|}
$\begin{array}{l}\text { Discordo } \\
\text { parcialmente }\end{array}$ \\
\end{tabular} & 9 & $6,3 \%$ \\
\hline & Não sei responder & 67 & $47,6 \%$ \\
\hline \multirow{5}{*}{$\begin{array}{l}\text { Você concorda que uma } \\
\text { cárie dentária, gengivite e } \\
\text { um canal não tratado } \\
\text { podem causar problemas } \\
\text { associados ao coração? }\end{array}$} & $\begin{array}{l}\text { Concordo } \\
\text { totalmente }\end{array}$ & 43 & $30,1 \%$ \\
\hline & \begin{tabular}{|l|} 
Concordo \\
parcialmente
\end{tabular} & 15 & $10,5 \%$ \\
\hline & \begin{tabular}{|l|} 
Discordo \\
totalmente
\end{tabular} & 9 & $6,3 \%$ \\
\hline & \begin{tabular}{|l|} 
Discordo \\
parcialmente
\end{tabular} & 4 & $2,8 \%$ \\
\hline & Não sei responder & 72 & $50,3 \%$ \\
\hline \multirow{5}{*}{$\begin{array}{l}\text { Você concorda que uma a } \\
\text { cárie dentária está } \\
\text { associada a quadros de } \\
\text { sinusite? }\end{array}$} & $\begin{array}{l}\text { Concordo } \\
\text { totalmente }\end{array}$ & 28 & $19,6 \%$ \\
\hline & \begin{tabular}{|l|l}
$\begin{array}{l}\text { Concordo } \\
\text { parcialmente }\end{array}$ \\
\end{tabular} & 20 & $14 \%$ \\
\hline & \begin{tabular}{|l}
$\begin{array}{l}\text { Discordo } \\
\text { totalmente }\end{array}$ \\
\end{tabular} & 9 & $6,3 \%$ \\
\hline & \begin{tabular}{|l|} 
Discordo \\
parcialmente
\end{tabular} & 8 & $5,6 \%$ \\
\hline & Não sei responder & 78 & $54,5 \%$ \\
\hline \multirow{5}{*}{$\begin{array}{l}\text { Você concorda que uma } \\
\text { cárie dentária, gengivite e } \\
\text { um canal não tratado } \\
\text { podem causar problemas } \\
\text { associados ao cérebro? }\end{array}$} & $\begin{array}{l}\text { Concordo } \\
\text { totalmente }\end{array}$ & 32 & $22,4 \%$ \\
\hline & \begin{tabular}{|l|} 
Concordo \\
parcialmente
\end{tabular} & 21 & $14,7 \%$ \\
\hline & \begin{tabular}{|l}
$\begin{array}{l}\text { Discordo } \\
\text { totalmente }\end{array}$ \\
\end{tabular} & 9 & $6,3 \%$ \\
\hline & \begin{tabular}{|l|} 
Discordo \\
parcialmente
\end{tabular} & 8 & $5,6 \%$ \\
\hline & Não sei responder & 73 & $51 \%$ \\
\hline \multirow{5}{*}{$\begin{array}{l}\text { Você concorda que uma } \\
\text { cárie dentária, gengivite e } \\
\text { um canal não tratado } \\
\text { podem levar a processos } \\
\text { mórbidos em r casos } \\
\text { evoluídos? }\end{array}$} & $\begin{array}{l}\text { Concordo } \\
\text { totalmente }\end{array}$ & 52 & $36,4 \%$ \\
\hline & \begin{tabular}{|l|}
$\begin{array}{l}\text { Concordo } \\
\text { parcialmente }\end{array}$ \\
\end{tabular} & 17 & $11,9 \%$ \\
\hline & \begin{tabular}{|l}
$\begin{array}{l}\text { Discordo } \\
\text { totalmente }\end{array}$ \\
\end{tabular} & 6 & $4,2 \%$ \\
\hline & \begin{tabular}{|l|}
$\begin{array}{l}\text { Discordo } \\
\text { parcialmente }\end{array}$ \\
\end{tabular} & 4 & $2,8 \%$ \\
\hline & Não sei responder & 64 & $44,8 \%$ \\
\hline
\end{tabular}

Em relação aos testes estatísticos para avaliar o nível de conhecimento nos diferentes grupos, o primeiro teste estatístico foi em 
relação aos gêneros, onde a média de acerto entre as mulheres foi maior que os homens (Tabela 3). Porém, segundo 0 teste-t independente, essas médias relacionadas aos gêneros não são significativas mostrando assim que não houve significância de acerto entre homens e mulheres $(p>0,05)$.

Tabela 3. Teste estatístico avaliando os acertos por gêneros $(n=143)$, Cajazeiras - PB, Brasil

\begin{tabular}{l|l|l|l|l}
\hline Variáveis & Número & Média de Acertos & Desvio Padrão & Valor de P \\
\hline Feminino & 120 & 7,4667 & 2,87830 & 0,032 \\
\hline Masculino & 23 & 5,9565 & 3,91359 & 0,032 \\
\hline Fonte: Dados da Pesquisa
\end{tabular}

O segundo teste estatístico buscou procurar se houve relevância de acertos entre alunos dos cursos da área de saúde e os alunos dos cursos da área de exatas. O teste-t independente demonstrou que não houve relevância entre esses dois grupos $(p>0,05)$, apesar de alunos da área de saúde terem obtido um média de acertos maior que alunos da área de exatas (Tabela 4).

Tabela 4. Teste estatístico avaliando os acertos por área de graduação $(n=143)$, Cajazeiras - PB, Brasil

\begin{tabular}{l|l|l|l|l}
\hline Variáveis & Número & Média de Acertos & Desvio Padrão & Valor de P \\
\hline AAS & 121 & 7,3554 & 3,04374 & 0,236 \\
\hline AAE & 22 & 6,5000 & 3,39116 & 0,236 \\
\hline \multicolumn{2}{l}{ Legenda: AAS Alunos da área de Saúde; AAE = Alunos da área de Exatas }
\end{tabular}

Fonte: Dados da Pesquisa

O terceiro teste estatístico teve o intuito de analisar os acertos dos participantes da pesquisa em uma perspectiva socioeconômica. Os universitários com renda de até dois salários mínimos foram considerados como "renda inferior", já os universitários com renda de 3 a 10 salários mínimos, foram considerados como "renda superior". O teste-t independente mostrou que na população dessa pesquisa não ocorreu diferenças relevantes em relação a quantidade de acertos ( $p>0,05)$. Entanto, é possível notar pela média de acertos, que alunos com renda superior, tiveram uma pequena vantagem em relação aos de renda inferior (Tabela 5).

Tabela 5. Teste estatístico avaliando os acertos relacionados à renda financeira $(n=143)$, Cajazeiras - PB, Brasil

\begin{tabular}{l|l|l|l|l}
\hline Variáveis & Número & Média de Acertos & Desvio Padrão & Valor de P \\
\hline RI & 121 & 7,3554 & 3,04374 & 0,236 \\
\hline RS & 22 & 6,5000 & 3,39116 & 0,236 \\
\hline Legenda: RI = Renda Inferior; RS = Renda Superior \\
Fonte: Dados da Pesquisa
\end{tabular}

O terceiro e último questionário, tinha a principal finalidade de investigar o nível de acesso a informação por parte dos universitários. Para isso, foram feitas duas perguntas referentes a esse assunto. A primeira delas indagava se os alunos tinham facilidade ao aceso a informações referente a saúde bucal, onde 105 dos entrevistados (73,4\%) respondeu que sim, contra apenas $38(26,6 \%)$ que respondeu que não. Já a segunda pergunta, questionava se os alunos recebiam instruções de higiene oral do cirurgião-dentista durante $\mathrm{o}$ atendimento odontológico. Mais da metade respondeu que sim $(78,3 \%)$, mostrando um bom percentual de acesso à informação e cuidados com saúde bucal (Gráficos 1 e 2).

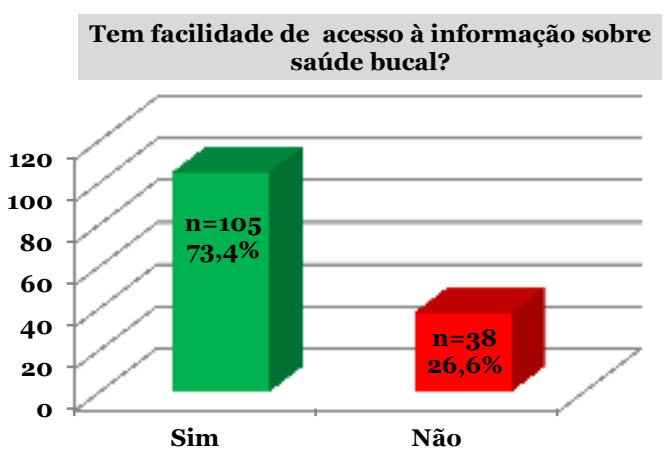

Gráfico 1. Distribuição dos acadêmicos quanto à facilidade de acesso à informação sobre saúde bucal.

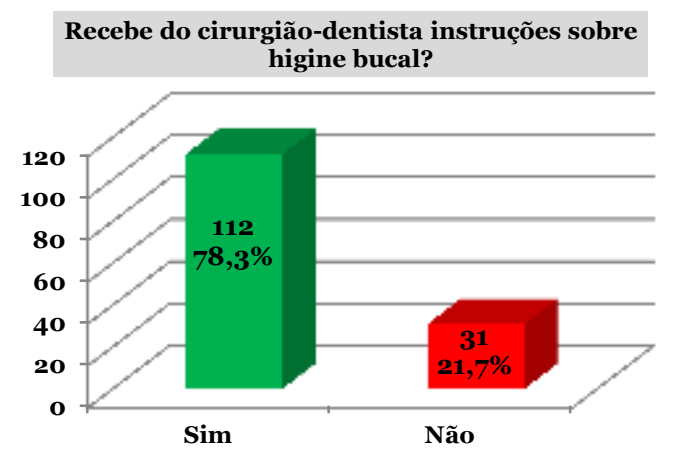

Gráfico 2. Distribuição dos acadêmicos quanto à facilidade de acesso à informação sobre saúde bucal.

DISCUSSÃO

Os resultados obtidos demonstram que a maioria dos universitários participantes da pesquisa apresenta uma boa percepção de saúde bucal, possivelmente esse dado se deve pelo fato dos entrevistados terem uma boa educação em saúde bucal que está diretamente associada ao grau de escolaridade dos alunos envolvidos. Em uma pesquisa realizada com pacientes atendidos em uma clínica odontológica, constou-se que quanto maior era o nível de escolaridade do indivíduo, maior era seu conhecimento referente a saúde bucal ${ }^{9}$.

No que diz respeito aos resultados dos dados socioeconômicos, a maioria dos universitários responderam que sua renda salarial é de apenas 1 salário mínimo. Segundo Hakeberg e Boman $^{10}$, o critério de baixa renda salarial é tido como um dos principais motivos de deficiência na saúde bucal e geral. Porém, os testes estatísticos realizados no presente trabalho não mostrou diferença significativa no número de acertos entre pessoas de renda baixa e renda alta.

Em relação aos resultados do questionário aplicado, a maioria dos alunos relatou ter conhecimento sobre o que é uma 
cárie dentária, uma gengivite e um tratamento de canal. Esses resultados são semelhantes a um estudo analítico realizado com professores de escolas públicas, onde $78,57 \%$ da população relatou que sabiam o que era a cárie ${ }^{11}$.

Em relação aos métodos utilizados para prevenir o surgimento de cáries e doenças gengivais, a maior parte dos universitários da pesquisa responderam que seria através de escovação, uso do fio dental, visitas regulares ao cirurgião-dentista e diminuição no consumo de açucares. Todas essas respostas corroboram com Cordeiro et al. ${ }^{12}$, em que $97,4 \%$ da população entrevistada respondeu que escovação, uso de fio dental e visitas regulares ao cirurgião-dentista seriam as principais formas de prevenção.

Os universitários também foram questionados se já haviam recebido alguma informação sobre saúde bucal. A maioria também respondeu que sim, informando que as redes sociais, palestras, através do cirurgiãodentista, em postos de saúde, escolas e na clínica Odontológica da própria Faculdade Santa Maria foram as principais fontes de informação, sendo 0 cirurgião-dentista 0 principal vetor de promoção de saúde, segundo os alunos da pesquisa. A Faculdade Santa Maria também entra como um meio de divulgação importante na educação em saúde bucal dos alunos entrevistados, visto que muitos relataram que a clínica Odontológica e os estudantes de Odontologia contribuíram muito com esse quesito.

Os resultados ainda mostraram que quando foi indagado se a cárie dentária, gengivite e infecção endodôntica poderiam ocasionar na formação de conteúdo purulento ou em uma internação hospitalar, a maioria dos participantes marcou a opção que dizia concordar totalmente, mostrando assim que a população da pesquisa apresenta um bom conhecimento relacionado a esses processos evolutivos de uma infecção odontogênica. Isso é bastante positivo, principalmente porque essas infecções tem seus graus de ascensão e disseminação pelo corpo, principalmente quando se tem a formação de conteúdo purulento Peterson et al. ${ }^{13}$.

Entanto, apesar dos alunos terem uma boa percepção de saúde bucal, nas ultimas perguntas do questionário, os resultados mostraram que não foi $100 \%$ satisfatório. Nas questões sobre cárie dentária, gengivite e infecção endodôntica poderem causar problemas associados ao coração e/ou cérebro, a maioria dos participantes não sabia responder. Esse dado é relativamente preocupante, pois as infecções odontogênicas complexas são apontadas como um problema relevante de saúde pública ${ }^{14}$. Nos estágios onde essa infecção atinge órgãos importantes, como o coração e o cérebro, o paciente pode ter um quadro de envolvimento das vias aérea e trombose do seio caverno, podendo causar até a morte ${ }^{15}$.

O motivo dos universitários não terem conhecimento dessas condições pode estar ligado a falta de informações aprofundadas em saúde bucal. Segundo Roberto et al. ${ }^{16}$, a falta de informações mais pontuais em saúde bucal faz com que o paciente tenha falhas no seu processo de educação em saúde e muitas vezes acabe negligenciando a procura por procedimentos odontológicos, buscando-os apenas em momentos críticos. Esses dados pontuam a importância de se abordar, de forma mais aprofundada, conteúdos relacionados a saúde bucal.

Em relação à realização da pesquisa, foi possível observar várias limitações durante a coleta de dados. Uma delas foi em relação aos questionários terem sido aplicados de forma online, pois muitas pessoas não estão adeptas a esse tipo de abordagem, dificultando assim a obtenção dos dados. Outro ponto importante a se frisar, seria a sugestão de novos trabalhos clínicos associados a esse, pois seria de grande relevância haver a associação desses dados epidemiológicos obtidos nessa pesquisa com os quadros clínicos dos pacientes obtidos em outras pesquisas.

CONCLUSÃO

Por meio dessa pesquisa pode-se observar que os universitários entrevistados da Faculdade Santa Maria, possuem uma boa percepção sobre infecções odontogênicas e seus processos de evolução. Os alunos demonstraram que possuem facilidade no acesso a informações sobre saúde bucal. Apesar dos alunos terem um bom conhecimento a respeito das infecções odontogênicas, esse deve ser aprofundado para garantir uma melhor promoção e prevenção na saúde odontológica. Esse aprofundamento pode ser feito por meio de palestras e/ou oficinas, discutindo temas que além da cárie dentária, mas também com enfoque em processos de evolução dessa doença e outras abordadas nessa pesquisa.

REFERÊNCIAS

1. Ogle OE. Odontogenic infections. Dent Clin North Am. 2017;61(2):235-52.

2. Rocha TAF, Cerqueira JDM, Carvalho ES. Infecções endodônticas persistente: causa, diagnóstico e tratamento. Rev Ciênc Méd Biol. 2018;17(1):78-83. 
3. Farah GJ, Quinto JHS, Farah IG, Chistoffo MT, Luppi CR. Estudo epidemiológico de pacientes portadores de infecção do complexo bucomaxilofacial tratados no Hospital Universitário de Maringá: estudo retrospectivo ao longo de 8 anos. RFO UPF. 2018;23(3): 280-83.

4. Oliveira RL, Raffaele RM, Baldo ME, Gaetti Jardim EC. Abscesso cerebral e infecção odontogênica. Rev Bras Ter Intensiva. 2020; 32(1):161-62

5. Pak S, Cha D, Meyer C, Dee CM, Fershko A. Ludwig's Angina. Cureus. 2017;9(8):e1588.

6. Mialhe FL, Silva CMC. A educação em saúde e suas representações entre alunos de um curso de Odontologia. Ciênc Saúde Colet. 2011;16 (1):1555-61

7. Silva CHF, Carneiro SV, Melo EAC, Dantas ES. Avaliação dos efeitos da educação em saúde sobre o conhecimento e comportamento de higiene bucal de escolares. Rev bras cienc saúde. 2019;23(2):215-22.

8. Lakatos EM, Marconi MA. Fundamentos da Metodologia Científica. 7. ed. São Paulo: Atlas; 2010.

9. Chou TTA, Ferreira NS, Kubo $\mathrm{CH}$, Silva EG, Huhtala MFR, Gonçalves SEP et al. Avaliação do conhecimento e comportamento dos pacientes em tratamento odontológico em relação à cárie, doença periodontal e higiene bucal. RPG Rev Pós Grad. 2011;18(3):140-47

10. Hakeberg M, Boman UW. Self-reported oral and general health in relation to socioeconomic position. BMC Public Health. 2018;18(63): 123-30

11. Santos MO, Casotti CA, Queiroz APDG, Carneiro JAO, Uemura TF. Conhecimento e percepção sobre saúde bucal de professores e pré-escolares de um município baiano. RFO UPF. 2015;20(2):171-78

12. Cordeiro GA, Junior MFS, Daroz LGD, Daroz CBS. Conhecimento sobre cárie dentária entre docentes do ensino médio. Rev ABENO. 2019; 19(3):133-43

13. Pinheiro HHC, Cardoso DG, Araújo MVA, Araújo IC. Avaliação do nível de conhecimento sobre saúde bucal dos professores da creche Sorena, Belém, Pará. J Health Sci Inst. 2005; 23(4):297-303.

14. Matthew TJH, Hussein A. Atypical cavernous sinus thrombosis: a diagnosis challenge and dilemma. Cureus. 2018;10(12):3685

15. Martini MZ, Uyeda DKP, Neto OLM, Santos NA. Choque séptico por infecção odontogênica em paciente com diabetes melito tipo I. Rev Assoc Paul Cir Dent. 2015;69(2):190-93.
16. Roberto LL, Noronha DD, Souza TO, Miranda EJ, Martins AMEBL, de Paula AMB et al. Falta de acesso a informações sobre problemas bucais entre adultos: abordagem baseada no modelo teórico de alfabetização em saúde. Ciênc Saúde Colet. 2018;23(3):823-35.

\section{CONFLITO DE INTERESSES}

Os autores declaram não haver conflitos de interesse

AUTOR PARA CORRESPONDÊNCIA

José Klidenberg de Oliveira Júnior

Rua Raimunda Xavier de Sousa 40, 58804-430 São José, Sousa, PB - Brasil

E-mail: joseklidemberg@gmail.com
Submetido em 13/04/2021 Aceito em 16/07/2021 